OPEN ACCESS

Edited by:

Panagiotis Balermpas,

University Hospital Zürich, Switzerland

Reviewed by:

Amir Abdollahi,

German Cancer Research Center

(DKFZ), Germany

Aviram Mizrachi,

Rabin Medical Center, Israel

*Correspondence:

Paolo Boscolo-Rizzo

paolo.boscolorizzo@units.it

Elisabetta Fratta

efratta@cro.it

${ }^{+}$These authors have contributed equally to this work and share last authorship

Specialty section: This article was submitted to Head and Neck Cancer, a section of the journal

Frontiers in Oncology

Received: 01 October 2021 Accepted: 08 November 2021 Published: 25 November 2021

Citation:

Polesel J, Menegaldo A, Tirelli G, Giacomarra V, Guerrieri R, Baboci L,

Casarotto M, Lupato V, Fanetti G,

Boscolo-Rizzo P and Fratta E (2021)

Prognostic Significance of PD-L1

Expression In Patients With Primary

Oropharyngeal Squamous Cell

Carcinoma: A Meta-Analysis.

Front. Oncol. 11:787864.

doi: 10.3389/fonc.2021.787864

\section{Prognostic Significance of PD-L1 Expression In Patients With Primary Oropharyngeal Squamous Cell Carcinoma: A Meta-Analysis}

\author{
Jerry Polesel ${ }^{1}$, Anna Menegaldo ${ }^{2}$, Giancarlo Tirelli ${ }^{3}$, Vittorio Giacomarra ${ }^{4}$, \\ Roberto Guerrieri ${ }^{5}$, Lorena Baboci ${ }^{5}$, Mariateresa Casarotto ${ }^{5}$, Valentina Lupato ${ }^{4}$, \\ Giuseppe Fanetti ${ }^{6}$, Paolo Boscolo-Rizzo ${ }^{3 * t}$ and Elisabetta Fratta ${ }^{5 * t}$ \\ 1 Unit of Cancer Epidemiology, Centro di Riferimento Oncologico di Aviano (CRO), IRCCS, Aviano, Italy, ${ }^{2}$ Unit of \\ Otolaryngology, AULSS 2 - Marca Trevigiana, Treviso, Italy, ${ }^{3}$ Department of Medical, Surgical and Health Sciences, \\ Section of Otolaryngology, University of Trieste, Trieste, Italy, ${ }^{4}$ Division of Otolaryngology, General Hospital "S. Maria \\ degli Angeli", Pordenone, Italy, ${ }^{5}$ Division of Immunopathology and Cancer Biomarkers, Centro di Riferimento Oncologico \\ di Aviano (CRO), IRCCS, Aviano, Italy, ${ }^{6}$ Division of Radiotherapy, Centro di Riferimento Oncologico di Aviano (CRO), \\ IRCCS, Aviano, Italy
}

Background: At present, the prognostic significance of programmed cell death receptor ligand 1 (PD-L1) expression in oropharyngeal squamous cell carcinoma (OPSCC) patients is still controversial. In this study, we aim to synthesize relevant studies that have assessed the prognostic value of PD-L1 in patients with primary OPSCC treated according to the current standard-of-care.

Methods: A systematic search of Medline/PubMed, Cochrane, Embase, Web of Science, and Scopus was conducted to define the prognostic role of PD-L1 expression in OPSCC. All studies published before July 31, 2021 were screened. Summary hazard ratios (sHR) with 95\% confidence intervals (Cls) were calculated using a random-effects model.

Results: A total of 1522 OPSCC patients from 12 studies were included. PD-L1 expression in OPSCC tumor cells (TCs) was significantly associated with longer overall survival (sHR=0.63, 95\% $\mathrm{Cl}$ 0.50-0.79), and progression-free survival (sHR=0.62, 95\% Cl 0.49-0.79). A benefit in survival was also observed in PD-L1-positive OPSCC patients who underwent surgery (sHR=0.34, 95\% Cl 0.18-0.65). Finally, although PD-L1-positive expression was related to better outcomes both in HPV-negative and HPV-positive OPSCC, the difference reached the statistical significance only in the HPV-positive subgroup (sHR=0.37, 95\% Cl 0.19-0.73). No heterogeneity emerged between studies for all considered outcomes, with $R^{2}$ ranging from $0 \%$ for progression-free survival to $11 \%$ for overall survival. 
Conclusions: PD-L1 expression on TCs associated with improved survival in OPSCC. In particular, HPV-positive OPSCC most benefited from PD-L1 expression when compared to the PD-L1 negative counterpart. Thus, PD-L1 might represent a useful biomarker to stratify prognosis in OPSCC in addition to HPV status.

Keywords: oropharyngeal squamous cell carcinoma, head and neck squamous cell carcinoma, PD-L1, HPV, prognostic biomarkers

\section{INTRODUCTION}

Despite its high immunogenicity, tumor microenvironment (TME) of both primary and recurrent head and neck squamous cell carcinoma (HNSCC) is associated with pronounced immunosuppressive activity (1). Immunosuppression creates an advantageous environment for HNSCC cells, that can evade tumor immune surveillance through various strategies, including the recruitment of suppressive cell populations, release of tumorderived soluble immunosuppressive factors, up-regulation of immune checkpoint inhibitors (ICI), and impaired costimulatory signaling (2).

At present, the up-regulation of the transmembrane glycoprotein programmed death ligand 1 (PD-L1) (3) is among the most potent and thoroughly investigated strategy implemented by tumor cells (TCs) and TME to suppress cellular immune responses. Once expressed, PD-L1 binds to the programmed death 1 ( $\mathrm{PD}-1)$, a negative co-stimulatory receptor that inhibits the activation of a wide range of immune cells, including peripherally activated T cells, B cells, monocytes, natural killer cells, and certain dendritic cells, thus playing a crucial role in the maintenance of immune tolerance of selfantigens $(4,5)$. Blocking these ICI has become an important direction of immunotherapy with antibodies targeting PD-1 or PD-L1 being currently approved for the treatment of multiple cancers including HNSCC (6). In 2016, the PD-1 inhibitors pembrolizumab and nivolumab have been approved by the Food and Drug Administration (FDA) for use in patients with recurrent or metastatic (R/M) HNSCC who progressed on standard platinum-based therapy (7). More recently, FDA extended the indications for pembrolizumab monotherapy in the first-line treatment of R/M HNSCC patients whose tumor and/or immune cells expressed PD-L1 with a Combined Positive Score (CPS) $\geq 1$ (8). Therefore, the ICI-based therapy blockade has been rapidly progressing in HNSCC, with a survival benefit in approximately $20-30 \%$ of patients (9-11).

Among HNSCC, the TME of the subset of oropharyngeal SCC (OPSCC) caused by human papilloma virus (HPV) is more enriched in immune cells than the HPV-negative counterpart, harbors higher levels of immune activation, and is characterized by the presence of cytotoxic T lymphocytes (CTL) specifically directed against HPV16 E6 and E7 oncoproteins (12, 13). Furthermore, a more pronounced expression of the immunoinhibitory receptors including CTLA-4 and PD-1 has been observed in HPV-positive OPSCC $(14,15)$. Thus, a highly immunogenic and more strongly immune infiltrated TME seems to be countered by a greater involvement of immunosuppressive strategies. These observations suggest that HPV-positive patients are more likely to benefit from anti-PD-1/PD-L1 therapy respect to HPV-negative ones. However, the current standard-of-care treatment for patients with primary OPSCC is still surgery +/adjuvant (chemo)radiotherapy or up-front (chemo)radiation with the last being a preferred option in several cases (16).

Recent studies have showed that radiotherapy can activate tumor-specific immune responses and these responses may be modulated by immune landscape of the TME (17). Moreover, PD-1 and PD-L1 expression was observed to predict radiosensitivity in HNSCC (18). Therefore, in addition to evaluating PD-1/PD-L1 expression as predictor of response to immunotherapy, there has been equally interest in evaluating the prognostic role of PD-1/PD-L1 in patients with primary OPSCC treated according to the current standard-of-care. A recent metaanalysis reported that HNSCC patients expressing PD-L1 may have a better tumor response and overall survival (OS) irrespective of their HPV status (19). However, data regarding the prognostic role of PD-L1 in HPV-positive OPSCC are still limited and controversial. In fact, although many studies have found that PD-L1 expression on TCs was associated with favorable prognosis of HPV-positive OPSCC patients (20-27), others found no association with prognosis or even a reverse relationship (28-35). Notably, the diverging results found in these studies may possibly depend on the use of different anti PD-L1 antibodies and cut-off values for determining PDL1 positivity.

Thus, given the inconsistency and inconclusive findings of the published data, we carried out an up-to-date systematic review and meta-analysis to determine whether PD-L1 expression affects OS, progression-free survival (PFS) and/or loco-regional control (LRC) of patients with primary HPV-positive and HPVnegative OPSCC treated according to the current standardof-care.

\section{MATERIALS AND METHODS}

\section{Ethics Statement}

Data from this meta-analysis were based on previous published papers; therefore, this study did not require ethical approval or patient consent.

\section{Outcome Measures}

The primary outcome of this meta-analysis was to investigate the prognostic value of PD-L1 for OS (defined as the time from diagnosis or initiation of treatment to patient death, irrespective of 
cause), PFS (defined as the time from diagnosis or initiation of treatment until tumor recurrence/progression or any-cause death), and LRC (defined as the time from diagnosis or initiation of treatment to the first loco-regional event) in patients treated by upfront surgery or (chemo)radiotherapy for OPSCC.

The secondary endpoint was the prognostic significance of PD-L1 on the above clinical outcome variables stratifying patients by type of treatment, upfront surgery or upfront (chemo) radiotherapy, and HPV status, as detected by $\mathrm{p} 16$ immunohistochemistry (IHC), HPV-DNA in situ hybridization or PCR.

\section{Search Strategy}

This systematic review and meta-analysis were conducted following the preferred reporting items for systematic reviews and meta-analyses (PRISMA) checklist. Medline/PubMed (via Ovid), Cochrane, Embase (via Ovid), Web of Science (Core Collection) and Scopus were searched from inception through the end of July 2021. The research was conducted according to PRISMA criteria (36). The following keyword search was conducted: "head and neck" OR "facial" OR "mouth" OR "oral cavity" OR "oropharyngeal" OR "pharyngeal” OR "oropharynx" OR "pharynx" AND "squamous cell carcinoma*" OR "carcinoma " OR "tumor*” OR "cancer*” OR "neoplasm»" AND "PD-L1" OR "B7-H1” OR “CD274” AND "prognosis" OR "risk" OR "recurrence ${ }^{\star}$ ” OR "mortality” OR "survival” OR "outcome*". The reference lists of articles included in this review as well as narrative reviews published in the last 10 years were also manually searched to minimize the risk of missing data. Two authors (PBR and EF) independently screened all titles and abstracts generated by the search and then evaluated the full texts of all the relevant articles identified against the inclusion criteria (Figure 1); a third author (JP) settled discordances when present. Any disagreement between the assessors on the suitability of articles for inclusion were tackled thorough discussion between assessors, or failing this, by referral to the other authors.

\section{Selection Criteria}

Studies were included in the analysis if they met the following criteria: 1) the study reported the prognostic role of PD-L1 in primary non-metastatic OPSCC; 2) the patients were treated according to the current standard of care; 3) studies in which PD-L1 expression was detected by IHC; 4) the study reported the association of PD-L1 and patient outcome with sufficient survival data to extract hazard ratios (HRs) and 95\% confidence intervals (CI). Non-English studies were excluded. Studies containing aggregated data or duplicated data from previously published work were excluded, as were review articles, case reports, editorials, and letters. Two authors (JP, PBR) independently assessed the quality of the included studies with the NewcastleOttawa Scale (37). Low-quality articles (Newcastle-Ottawa Scale (NOS) score $<4$ ) were also excluded.

\section{Data Extraction and Statistical Analysis}

The standard error of the log hazard ratio was derived from the $\log$ CIs. The summary HR (sHR) and corresponding 95\% CI were calculated according to random-effects models of DerSimonian and Laird (38), which incorporates both within-and betweenstudy variability, as a weighted average of the estimated HRs, by giving each study a weight proportional to its precision. Statistical heterogeneity among studies was evaluated using the $I^{2}$ and $\tau^{2}$ statistics (38). Influence analysis was performed when sHRs were estimated from five or more studies: sHR was calculated by omitting one study at a time. Publication bias was assessed through a funnel plot (39). The results of the meta-analysis were presented graphically using forest plots, plotting the individual paper and sHR with corresponding 95\% CI. Statistical significance was defined as $\mathrm{p}<0.05$ (two sided).

\section{RESULTS}

\section{Search Results and Study Characteristics}

A total of 2078 potentially relevant articles were identified with our initial search strategy. After screening the titles and abstracts of these articles, 2042 studies were excluded because they were deemed repetitive or unqualified. After reading 36 potentially eligible articles in detail, 13 studies met our inclusion criteria. However, since the study by Salomon et al. (27) excluded patients with HPV-negative OPSCC, thus introducing a potential selection bias, it was excluded from the analysis, thus leaving 12 articles in the final analysis $(17,20-25,28-30,32,34)$. No additional studies were obtained by checking the reference lists of these articles. Figure 1 presents a detailed diagram of the above screening process.

The characteristics of the included studies are reported in Table 1. The sample sizes of these studies ranged from 65 to 303 patients for a total of 1522 patients. Different cut-off values for the percentage of PD-L1-positive TCs were utilized in 12 studies $(17,20-25,27-30,34)$, ranging from $1 \%$ to $50 \%$, while one study used the Combined Positive Score (CPS) $\geq 1$ to define a PD-L1 positive OPSCC (32). The rates of PD-L1 positive OPSCC in the above studies ranged from $7.7 \%$ to $88.7 \%$ with overall 910 patients $(53.4 \%)$ being PD-L1 positive. With the exception of one study that only selected HPV positive OPSCC (27), all other studies included consecutive patient series selected regardless of HPV status.

\section{Quality Assessment}

All included studies reported a satisfactory quality (NewcastleOttawa Scale score $\geq 6$ ), with a median of 8 (Table 2). The most frequent sources of potential bias were specific selection criteria that could have impaired external validity (e.g., restriction to tonsillar cancer or to patients undergoing radiation therapy) and the lack of reporting information of the completeness of follow-up. Only two studies did not report multivariable estimates.

\section{PD-L1 Expression as a Biomarker of Outcomes}

All articles provided HRs and 95\% CIs of OS in OPSCC. As shown in Figure 2A, no evident heterogeneity was observed in these studies $\left(I^{2}=11 \% ; \mathrm{p}=0.34\right)$. The results revealed that positive PD-L1 expression was related to a significantly improved OS in patients 


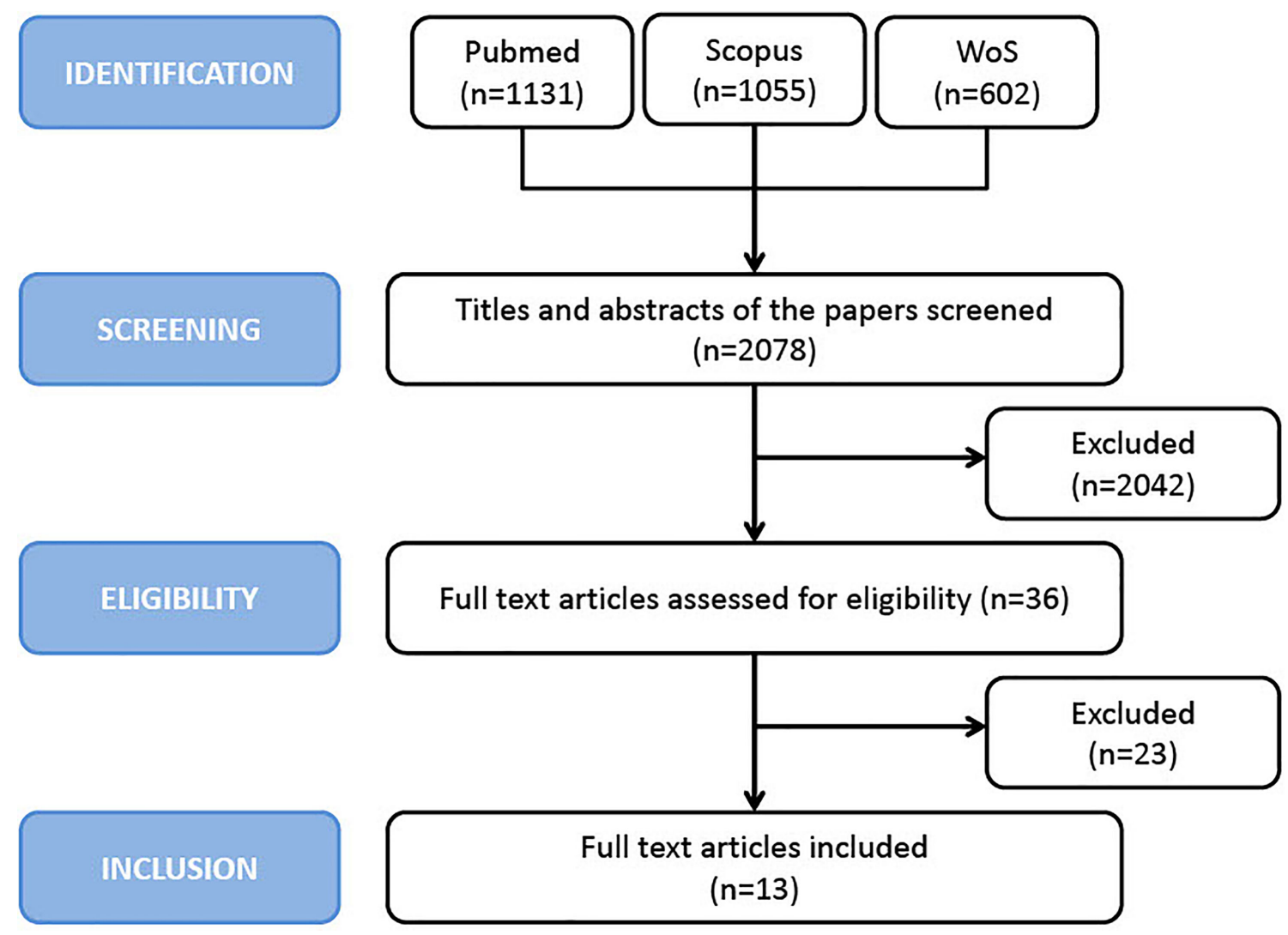

FIGURE 1 | PRISMA flow chart of study inclusion process.

TABLE 1 | Description of included studies.

\begin{tabular}{|c|c|c|c|c|c|c|}
\hline Study & Country & Enrolment & $\mathbf{n}$ & HPV-status & PD-L1 antibody & Cut-off for PD-L1 positivity \\
\hline Gurin (29) & Czech Republic & - & 65 & Pos/Neg & Clone 28-8 (Abcam) & $\mathrm{TC} \geq 5 \%$ \\
\hline Jeong (30) & Korea & 2006-2013 & 106 & Pos/Neg & Clone SP263 (Ventana) & $\mathrm{TC} \geq 50 \%$ \\
\hline Lilja-Fisher (32) & Denmark & 2000-2012 & 303 & Pos/Neg & Clone 22C3 (PharmDx) & $\mathrm{CPS} \geq 1$ \\
\hline Hong (22) & Australia & - & 214 & Pos/Neg & Clone E1L3N (CST) & $\mathrm{TC} \geq 1 \%$ \\
\hline Sato (26) & Japan & 2000-2016 & 137 & Pos/Neg & Clone E1L3N (CST) & $\mathrm{TC} \geq 5 \%$ \\
\hline Fukushima (21) & Japan & $2005-2016$ & 92 & Pos/Neg & Clone SP142 (Ventana) & $\mathrm{TC} \geq 1 \%$ \\
\hline Kwon (25) & South Korea & 1997-2010 & 79 & Pos/Neg & Clone SP142 (Ventana) & $\mathrm{TC} \geq 5 \%$ \\
\hline Steuer (24) & U.S.A. & 1994-2008 & 97 & Pos/Neg & $(\mathrm{CST})$ & $\mathrm{TC} \geq 1+$ \\
\hline Balermpas (28) & Germany & 2004-2012 & 98 & Pos/Neg & Clone E1L3N (CST) & $\mathrm{TC} \geq 5 \%$ \\
\hline De Meulenaere (20) & Belgium & 2004-2013 & 99 & Pos/Neg & Clone SP142 (Roche) & $\mathrm{TC} \geq 5 \%$ \\
\hline Hong (23) & Australia & - & 99 & Pos/Neg & Clone E1L3N (CST) & - \\
\hline Kim (24) & South Korea & 2002-2013 & 133 & Pos/Neg & B7H1 Clone 5H1 & $\mathrm{TC} \geq 20 \%$ \\
\hline
\end{tabular}

NOS, Newcastle-Ottawa Scale.

with OPSCC (sHR=0.63, 95\% CI: 0.50-0.79). Differently, only seven articles provided HRs and 95\% CIs for PFS in OPSCC (17, 20-23, 25, 29). As depicted in Figure 2B, there was no significant heterogeneity among these 7 articles $\left(I^{2}=0 \% ; \mathrm{p}=0.45\right)$. The metaanalysis indicated that positive $\mathrm{PD}-\mathrm{L} 1$ expression was related to greater PFS in patients with OPSCC $(\mathrm{sHR}=0.62$, 95\% CI: $0.49-$ 0.79). Finally, the association between PD-L1 expression and LRC was investigated in four studies $(22,23,29,32)$ and a significant heterogeneity was not detected among them $\left(I^{2}=48 \%\right.$; $\left.=0.12\right)$ (Figure 2C). Although a significant relationship did not emerged, results showed that LRC was improved in OPSCC patients that were $\mathrm{PD}-\mathrm{L} 1$ positive $(\mathrm{sHR}=0.92,95 \% \mathrm{CI}$ : $0.55-1.53)$.

\section{Impact of HPV Status}

The subgroup analysis based on HPV status showed that PD-L1-positive expression was related to better OS both in 
TABLE 2 | Quality assessment of included studies according to the Newcastle-Ottawa Scale (http://www.ohri.ca/programs/clinical_epidemiology/oxford.asp).

\begin{tabular}{|c|c|c|c|c|c|c|c|c|c|}
\hline \multirow[t]{2}{*}{ Study } & \multicolumn{4}{|c|}{ Selection } & \multirow[t]{2}{*}{ Comparability $^{a}$} & \multicolumn{3}{|c|}{ Outcome } & \multirow{2}{*}{$\begin{array}{c}\text { Total } \\
\text { Score } \\
(0-9)\end{array}$} \\
\hline & $\begin{array}{l}\text { Representativeness } \\
\text { of exposed cohort }\end{array}$ & $\begin{array}{l}\text { Selection } \\
\text { of non- } \\
\text { exposed } \\
\text { cohort }\end{array}$ & $\begin{array}{c}\text { Exposure } \\
\text { ascertainment }\end{array}$ & $\begin{array}{c}\text { Outcome } \\
\text { not present } \\
\text { prior to } \\
\text { exposure }\end{array}$ & & $\begin{array}{l}\text { Independent } \\
\text { assessment }\end{array}$ & $\begin{array}{l}\text { Adequacy of } \\
\text { follow-up } \\
\text { (median } \geq 24 \\
\text { months) }\end{array}$ & $\begin{array}{l}\text { Completeness } \\
\text { of follow-up } \\
\text { ascertainment }\end{array}$ & \\
\hline Gurin (29) & ○ & - & ○ & 0 & $\bigcirc$ & $\bullet$ & - & 0 & 6 \\
\hline Jeong (30) & 0 & ○ & 0 & 0 & $\bullet$ & ○ & ○ & 0 & 8 \\
\hline Lilja-Fisher (32) & O & - & - & 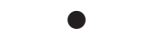 & ○० & ○ & 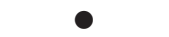 & 0 & 7 \\
\hline Hong (22) & 0 & $\bullet$ & $\bullet$ & 0 & $\bullet$ & 0 & 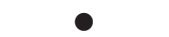 & 0 & 8 \\
\hline Sato (26) & $\bullet$ & - & - & 0 & 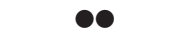 & 0 & 0 & 0 & 8 \\
\hline Fukushima (21) & 0 & - & - & $\bullet$ & $\bullet$ & $\bullet$ & 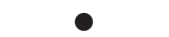 & $\bullet$ & 7 \\
\hline Kwon (25) & O & - & - & 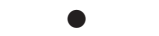 & 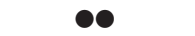 & 0 & 0 & 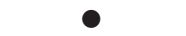 & 8 \\
\hline Steuer (24) & 0 & - & ○ & 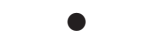 & $\bullet$ & 0 & 0 & 0 & 8 \\
\hline Balermpas (28) & O & - & - & 0 & ○० & 0 & ○ & 0 & 7 \\
\hline De Meulenaere (20) & - & - & - & ○ & O० & ○ & 0 & ○ & 7 \\
\hline Hong (23) & ○ & - & ○ & 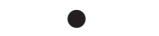 & ○० & ○ & 0 & 0 & 8 \\
\hline Kim (24) & 0 & $\bullet$ & ○ & 0 & $\bullet$ & 0 & 0 & 0 & 7 \\
\hline
\end{tabular}

${ }^{a} 1$ point for multivariable analyses; 1 point for stratification by HPV.

HPV-negative and HPV-positive OPSCC. However, few studies $(22,23,28,30,32)$ reported HRs and $95 \%$ CI for OS according to $\mathrm{HPV}$-status and the difference reached the statistical significance only in the subgroup of HPV-positive OPSCC ( $\mathrm{sHR}=0.37,95 \% \mathrm{CI}$ 0.19-0.73) (Figures 3A, B).

\section{Impact of Treatment}

The subgroup analysis based on treatment modality [upfront surgery or upfront (chemo)radiotherapy] revealed a better OS in PD-L1 positive tumors, regardless of treatment type (Figures 4A, B). Nevertheless, the effect was greater and significant only in the group treated with up-front surgery $(\mathrm{sHR}=0.34$, 95\% CI: $0.18-$ $0.65)$. Unfortunately, data available for the present analysis was limited since only six studies were included $(21,24,25,28$, 29, 32).

\section{Publication Bias and Sensitivity Analysis}

We inspected publication bias through a funnel plot. The result (Figure 5) indicated a lack of publication bias (test for asymmetry in funnel plot: $\mathrm{p}=0.89$ ). Furthermore, influence analysis was implemented by eliminating one by one studies to determine whether a single study could significantly influence the summary result. The results showed no relevant impact on the overall result by any study (Figure 6), with sHRs ranging from 0.59 by excluding Steuer et al. to 0.66 by excluding either Sato et al. (26) or Kwon et al. (25).

\section{DISCUSSION}

In the last years, several studies evaluated PD-L1 expression in HNSCC tissues $(40,41)$. Nevertheless, the relationship between PD-L1 expression and the prognosis of OPSCC patients was still controversial. Two meta-analyses conducted in 2017 and 2018 have not observed a prognostic effect of tumor PD-L1 positivity in patients diagnosed with OPSCC $(42,43)$. However, although seventeen studies were considered eligible by Li et al. in 2017, only two of them focused on OPSCC (44). In 2018, Yang et al. 2018 included three additional studies, which mainly comprised OPSCC, but a statistically significant benefit in OS was still not observed (43), thus suggesting these results might depend on the limited number of survival data. The results of this meta-analysis confirmed that, in patients diagnosed with OPSCC, PD-L1 expression was associated with a better OS and PSF, but not with LRC. Notably, higher PD-L1 expression was correlated with improved OS rates in other tumor types, including melanoma (45), colorectal cancer (46), Merkel-cell carcinoma (47), and endometrial cancer $(48,49)$.

Of interest, in our study, the favorable effect of PD-L1 was stronger for HPV-positive patients. In fact, subgroup analysis by HPV status showed a $60 \%$ reduction in mortality in patients who expressed PD-L1 compared to those who did not. Consistent with these data, PD-L1 levels were significantly higher in HPVpositive OPSCC, suggesting a relationship between HPV status and PD-L1 expression. Although HPV oncoproteins E5 and E6/ E7 were supposed to activate the PD-1/PD-L1 axis (50), further research is required to elucidate the association between HPV oncoproteins and PD-L1. In addition, since PD-L1 gets upregulated in response to tumor-infiltrating lymphocytes (TIL)derived cytokines, the increase in PD-L1 expression in HPVpositive OPSCC might be related to a more inflamed tumor microenvironment with recruitment of TILs, rather than to a direct causative effect of HPV on PD-L1 expression (51). This theory is in line with the evidence that abundant TILs and high PD-L1 expression in intratumoral immune cells identified subgroups of HPV-positive OPSCC patients with excellent outcomes (27). These observations might seem a paradox since TILs are supposed to mount anti-tumor immune responses whereas PD-L1 is assumed to impair their activity. Thus, the impression is that the expression of $\mathrm{PD}-1 / \mathrm{PD}-\mathrm{L} 1$ axis may be the reflection of the antitumor reactivity rather than a sign of immune exhaustion. Recent studies have indicated that evaluation of PD-L1 protein status could assist in the selection of patients with OPSCC who are candidates 


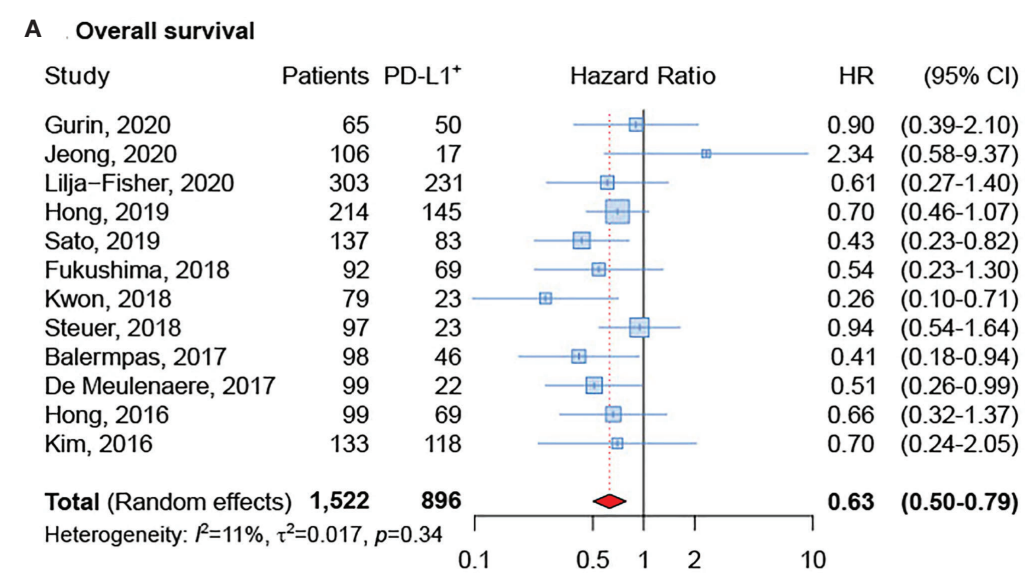

B Progression-free survival

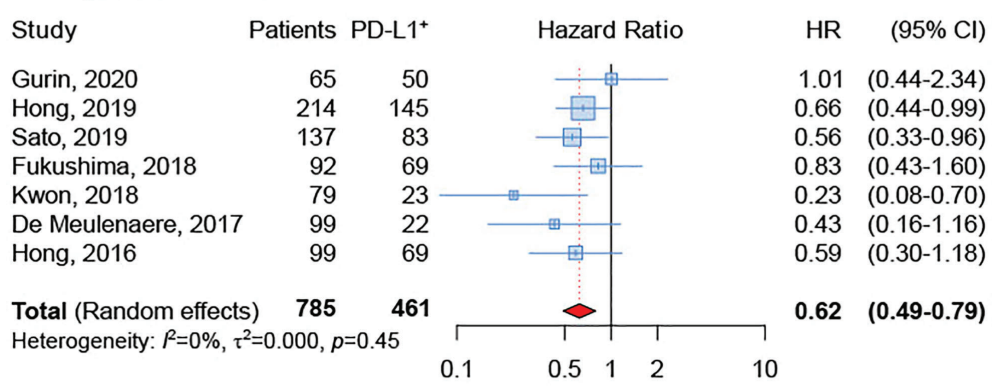

C Loco-regional control

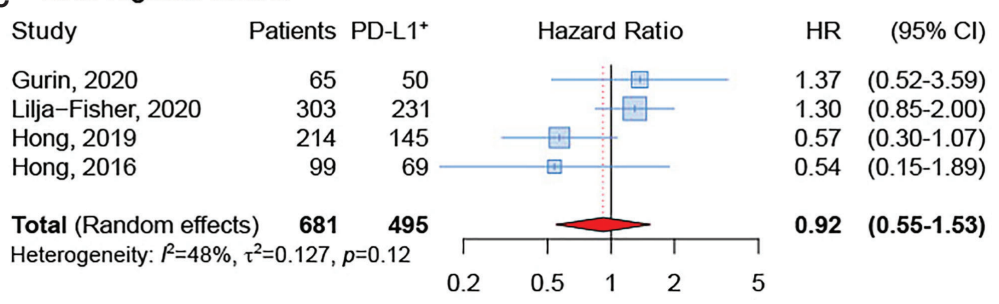

FIGURE 2 | Forest plot for the association between PD-L1 expression and overall survival (A), progression-free survival (B), and loco-regional control (C).

for ICI immunotherapy (52). However, the role of ICI in platinum-refractory recurrent/metastatic (R/M) HNSCC is still controversial with only a small fraction of patients experiencing clinical benefit from anti-PD-1 monotherapy or in combination with chemotherapy (53). On this ground, our findings would also support the use of antibodies targeting the PD-1/PD-L1 axis for these patients. Particularly, the tumor microenvironment of HPV-positive OPSCC, highly infiltrated by immune cells whose activity is blocked by the expression of ICI, makes these tumors ideal candidates for trials testing first-line ICI immunotherapy.

HPV-driven OPSCC have significantly better survival rates than tobacco and alcohol induced HNSCC (54). Since patients with HPV-driven cancer are younger, healthier and far more likely to survive their disease, long-term treatment-related toxicities are major issue in this population. With the aim to reduce toxicity while maintaining efficacy, treatment de- escalation strategies are currently investigated in several clinical trials. However, the only two phase III de-intensification trials failed to show the equivalence of the de-escalated treatment arm $(55,56)$. One possible explanation may lie in the fact that HPVpositive OPSCC is actually a heterogeneous disease. Interestingly, a subgroup of patients with confirmed HPV-driven OPSCC were shown to have an immunologically "colder" microenvironment and markedly poorer clinical outcome (57). Given that PD-L1 is able to stratify the prognosis in the group of HPV-positive tumors, it could be interesting to explore the opportunity of using PD-L1, possibly together with other clinical and molecular parameters, as a tool to improve the selection of patients for treatment de-intensification trials.

\section{Strengths and Limitations}

The strength of our study relies on the inclusion of very recent data, adding further precision to the evidence that PD-L1 


\section{A HPV-negative}

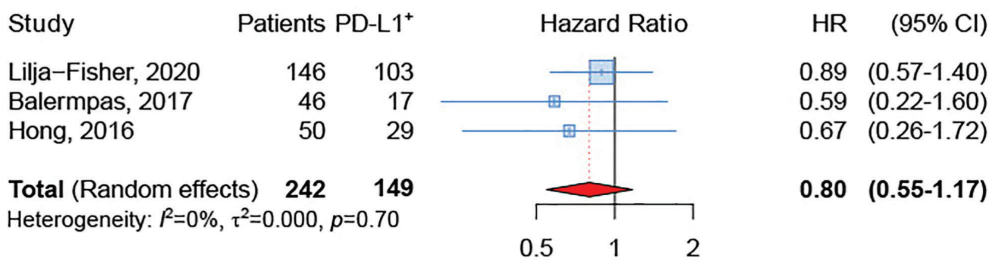

B HPV-positive

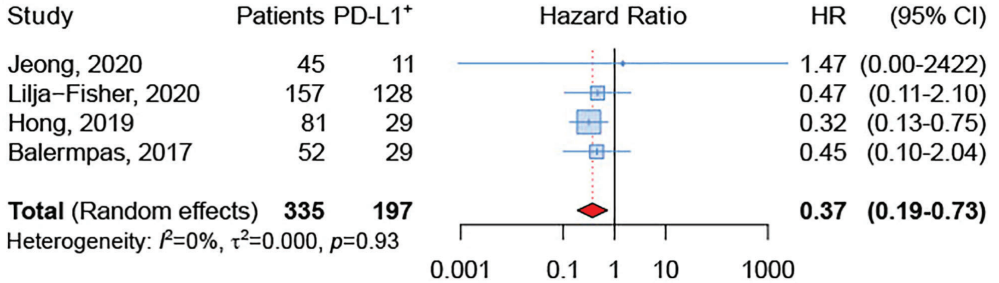

FIGURE 3 | Forest plot for the association between PD-L1 expression and overall survival in HPV-negative (A) and HPV-positive (B) tumors.

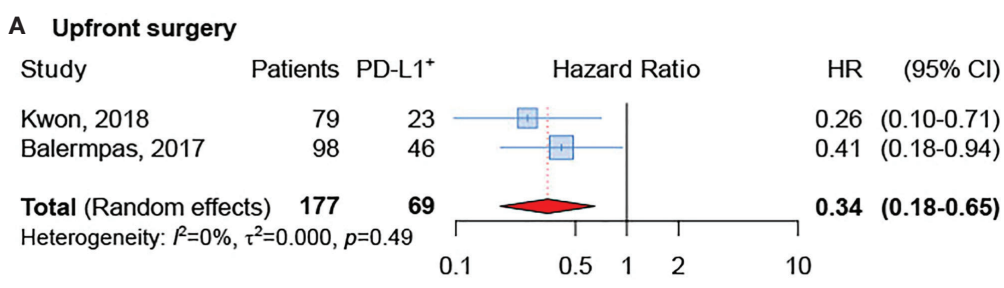

B Upfront radio-chemotherapy

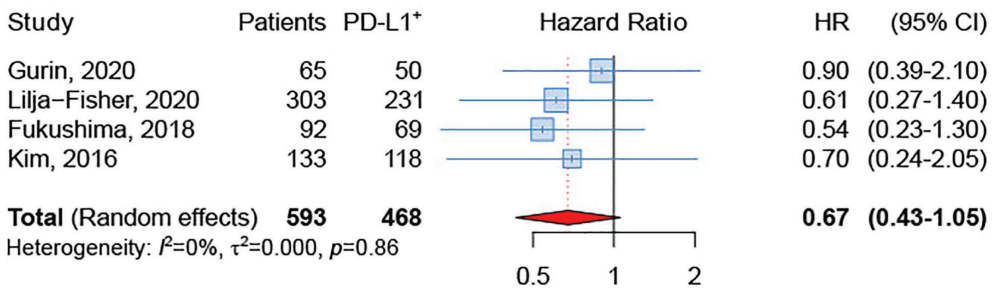

FIGURE 4 | Forest plot for the association between PD-L1 expression and overall survival in patients undergoing upfront surgery (A) or upfront radio-chemotherapy (B).

expression in TCs might be a favorable prognostic marker in OPSCC. However, several limitations exist in our meta-analysis. First, since the number of included studies was small for some outcomes and for sub-group analyses, bias may have been introduced. This occurred especially when the studies were greatly unbalanced in sample size, as summary estimates were more sensitive to single studies. Further, heterogeneity estimation was challenging in this context; in addition to imprecision, the $I^{2}$ statistic may also suffer of estimation bias (58), claiming for caution in its interpretation. Second, some information was not available in all included studies; for instance, data collected from published papers often lacked individual results for HPV status and/or treatment modality; hence, we failed to conduct a stratified analysis of the influence of PD-L1 expression on the response to surgery and/or radio-therapy in subgroups of HPV-positive and -negative OPSCC patients. In addition, since it has recently been shown that the level of PD-L1 expression was discrepant between primary HNSCC and corresponding distant metastases (59), only primary OPSCC have been considered. Third, this meta-analysis might not include studies that were not published due to negative results, which are often rejected or 


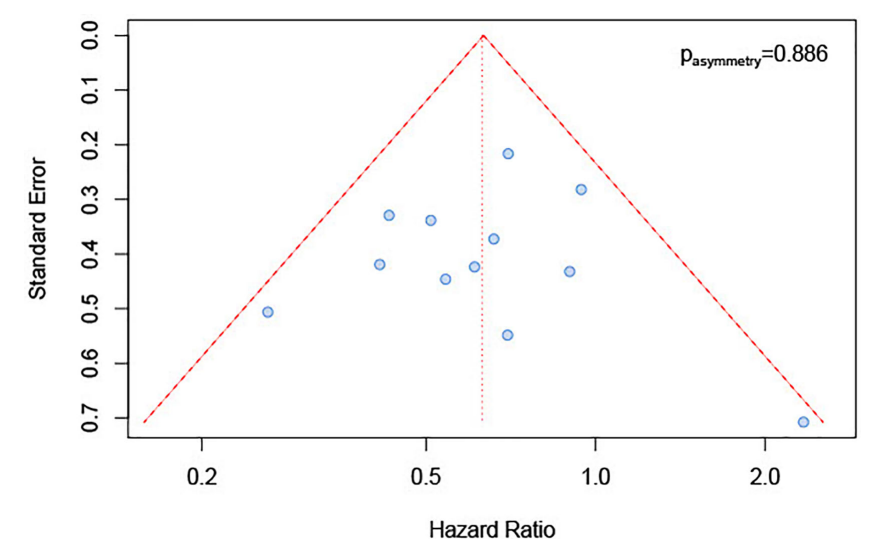

FIGURE 5 | Funnel plot for publication bias.

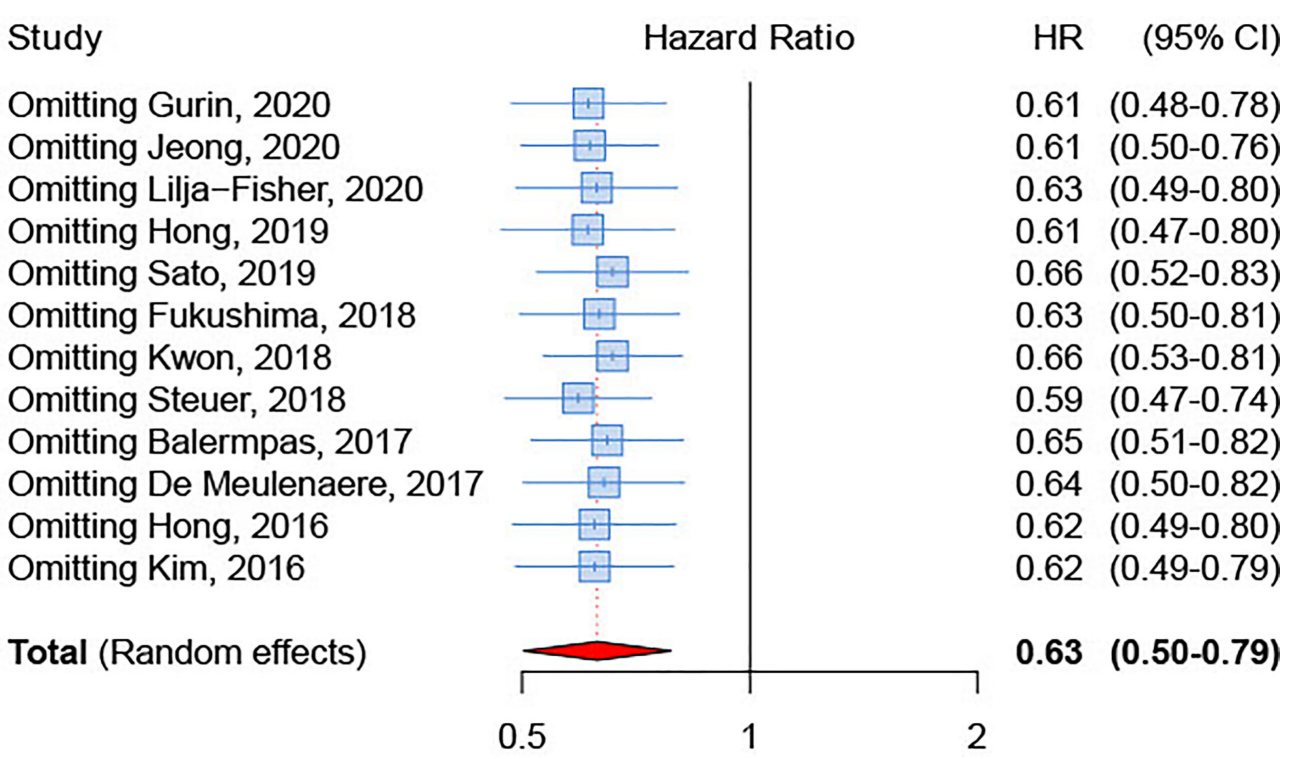

FIGURE 6 | Influence analysis on the association between PD-L1 expression and overall survival.

not even submit. Nonetheless, no publication bias emerged according to funnel plot inspection. Fourth, to compare OPSCC patient survival based on PD-L1 expression status might depend on the variability of IHC assay. In fact, accurate measurement of PD-L1 protein levels in FFPE tumor samples could be problematic due to antibodies discrepancy, differences in types of platforms used for the IHC staining and scoring criteria. A significant degree of intratumoral heterogeneity of PD-L1 expression might also represent an important limitation (60). Finally, the studies included in this meta-analysis have utilized a variety of techniques for determining the HPV status, including p16 IHC, HPV-DNA in situ hybridization or PCR. Hence, a standardized method for quantifying PD-L1 expression and determining HPV status is urgently needed.

\section{Conclusions}

The present meta-analysis showed that PD-L1 expression, as measured by IHC, was significantly associated with a better outcome in primary OPSCC treated according to the current standard of care. Particularly, HPV-positive OPSCC most benefited from PD-L1 expression with those expressing PD-L1 showing a reduction of $63 \%$ in the risk of death compared to the PD-L1 negative counterpart. Thus, PD-L1 might represent a useful biomarker, outside the context of ICI immunotherapy, to stratify prognosis in OPSCC in addition to HPV status. 


\section{DATA AVAILABILITY STATEMENT}

Publicly available datasets were analyzed in this study. This data can be found here: Data are available in the originally published articles.

\section{AUTHOR CONTRIBUTIONS}

JP, PB-R and EF designed the study and wrote the manuscript. AM, GT, VG, RG, LB, MC, VL and GF critically revised the manuscript. All authors contributed to the article and approved the submitted version.

\section{REFERENCES}

1. Watermann C, Pasternack H, Idel C, Ribbat-Idel J, Brägelmann J, Kuppler P, et al. Recurrent HNSCC Harbor an Immunosuppressive Tumor Immune Microenvironment Suggesting Successful Tumor Immune Evasion. Clin Cancer Res (2021) 27(2):632-44. doi: 10.1158/1078-0432.CCR-20-0197

2. Horton JD, Knochelmann HM, Day TA, Paulos CM, Neskey DM. Immune Evasion by Head and Neck Cancer: Foundations for Combination Therapy. Trends Cancer (2019) 5(4):208-32. doi: 10.1016/j.trecan.2019.02.007

3. Zhang Y, Zhang Z. The History and Advances in Cancer Immunotherapy: Understanding the Characteristics of Tumor-Infiltrating Immune Cells and Their Therapeutic Implications. Cell Mol Immunol (2020) 17(8):807-21. doi: 10.1038/s41423-020-0488-6

4. Jiang X, Wang J, Deng X, Xiong F, Ge J, Xiang B, et al. Role of the Tumor Microenvironment in PD-L1/PD-1-Mediated Tumor Immune Escape. Mol Cancer (2019) 18(1):10. doi: 10.1186/s12943-018-0928-4

5. Okazaki T, Honjo T. PD-1 and PD-1 Ligands: From Discovery to Clinical Application. Int Immunol (2007) 19(7):813-24. doi: 10.1093/intimm/dxm057

6. Sun L, Zhang L, Yu J, Zhang Y, Pang X, Ma C, et al. Clinical Efficacy and Safety of Anti-PD-1/PD-L1 Inhibitors for the Treatment of Advanced or Metastatic Cancer: A Systematic Review and Meta-Analysis. Sci Rep (2020) 10 (1):2083. doi: 10.1038/s41598-020-58674-4

7. Seiwert TY, Burtness B, Mehra R, Weiss J, Berger R, Eder JP, et al. Safety and Clinical Activity of Pembrolizumab for Treatment of Recurrent or Metastatic Squamous Cell Carcinoma of the Head and Neck (KEYNOTE-012): An Open-Label, Multicentre, Phase 1b Trial. Lancet Oncol (2016) 17(7):956-65. doi: 10.1016/S1470-2045(16)30066-3

8. Rischin D, Harrington KJ, Greil R, Soulieres D, Tahara M, Castro Gd, et al. Protocol-Specified Final Analysis of the Phase 3 KEYNOTE-048 Trial of Pembrolizumab (Pembro) as First-Line Therapy for Recurrent/Metastatic Head and Neck Squamous Cell Carcinoma (R/M HNSCC). J Clin Oncol (2019) 37(15_suppl):6000. doi: 10.1200/JCO.2019.37.15_suppl.6000

9. Burtness B, Harrington KJ, Greil R, Soulières D, Tahara M, de Castro GJr., et al. Pembrolizumab Alone or With Chemotherapy Versus Cetuximab With Chemotherapy for Recurrent or Metastatic Squamous Cell Carcinoma of the Head and Neck (KEYNOTE-048): A Randomised, Open-Label, Phase 3 Study. Lancet (2019) 394(10212):1915-28. doi: 10.1016/S0140-6736(19) 32591-7

10. Cohen EEW, Soulières D, Le Tourneau C, Dinis J, Licitra L, Ahn MJ, et al. Pembrolizumab Versus Methotrexate, Docetaxel, or Cetuximab for Recurrent or Metastatic Head-and-Neck Squamous Cell Carcinoma (KEYNOTE-040): A Randomised, Open-Label, Phase 3 Study. Lancet (2019) 393(10167):156-67. doi: 10.1016/S0140-6736(18)31999-8

11. Ferris RL, Blumenschein GJr., Fayette J, Guigay J, Colevas AD, Licitra L, et al. Nivolumab vs Investigator's Choice in Recurrent or Metastatic Squamous Cell Carcinoma of the Head and Neck: 2-Year Long-Term Survival Update of CheckMate 141 With Analyses by Tumor PD-L1 Expression. Oral Oncol (2018) 81:45-51. doi: 10.1016/j.oraloncology.2018.04.008

12. Albers A, Abe K, Hunt J, Wang J, Lopez-Albaitero A, Schaefer C, et al. Antitumor Activity of Human Papillomavirus Type 16 E7-Specific T Cells

\section{FUNDING}

This work was supported by Ministero della Salute Ricerca Corrente and 5x1000 Intramural Grant from Centro di Riferimento Oncologico Aviano (CRO) IRCCS.

\section{ACKNOWLEDGMENTS}

We thank Mrs Luigina Mei, Unit of Cancer Epidemiology Centro di Riferimento Oncologico Aviano (CRO) IRCCS, for editorial assistance. We are deeply thankful to Prof. Emmanouil Fokas, Dr. Jens von der Grün and group for provision original data from the manuscript Balermpas et al.

Against Virally Infected Squamous Cell Carcinoma of the Head and Neck. Cancer Res (2005) 65(23):11146-55. doi: 10.1158/0008-5472.CAN-05-0772

13. Partlová S, Bouček J, Kloudová K, Lukešová E, Zábrodský M, Grega $M$, et al. Distinct Patterns of Intratumoral Immune Cell Infiltrates in Patients With HPV-Associated Compared to Non-Virally Induced Head and Neck Squamous Cell Carcinoma. Oncoimmunology (2015) 4(1):e965570. doi: 10.4161/21624011.2014.965570

14. Carrero I, Liu HC, Sikora AG, Milosavljevic A. Histoepigenetic Analysis of HPV - and Tobacco-Associated Head and Neck Cancer Identifies Both Subtype-Specific and Common Therapeutic Targets Despite Divergent Microenvironments. Oncogene (2019) 38(19):3551-68. doi: 10.1038/s41388018-0659-4

15. Mandal R, Şenbabaoğlu Y, Desrichard A, Havel JJ, Dalin MG, Riaz N, et al. The Head and Neck Cancer Immune Landscape and Its Immunotherapeutic Implications. JCI Insight (2016) 1(17):e89829. doi: 10.1172/jci.insight.89829

16. Bigelow EO, Seiwert TY, Fakhry C. Deintensification of Treatment for Human Papillomavirus-Related Oropharyngeal Cancer: Current State and Future Directions. Oral Oncol (2020) 105:104652. doi: 10.1016/j.oraloncology.2020.104652

17. Sato H, Okonogi N, Nakano T. Rationale of Combination of Anti-PD-1/PDL1 Antibody Therapy and Radiotherapy for Cancer Treatment. Int J Clin Oncol (2020) 25(5):801-9. doi: 10.1007/s10147-020-01666-1

18. Lyu X, Zhang M, Li G, Jiang Y, Qiao Q. PD-1 and PD-L1 Expression Predicts Radiosensitivity and Clinical Outcomes in Head and Neck Cancer and Is Associated With HPV Infection. J Cancer (2019) 10(4):937-48. doi: 10.7150/ jca.27199

19. Patel JJ, Levy DA, Nguyen SA, Knochelmann HM, Day TA. Impact of PD-L1 Expression and Human Papillomavirus Status in Anti-PD1/PDL1 Immunotherapy for Head and Neck Squamous Cell Carcinoma-Systematic Review and Meta-Analysis. Head Neck (2020) 42(4):774-86. doi: 10.1002/ hed.26036

20. De Meulenaere A, Vermassen T, Aspeslagh S, Deron P, Duprez F, Laukens D, et al. Tumor PD-L1 Status and CD8(+) Tumor-Infiltrating T Cells: Markers of Improved Prognosis in Oropharyngeal Cancer. Oncotarget (2017) 8 (46):80443-52. doi: 10.18632/oncotarget.19045

21. Fukushima Y, Someya M, Nakata K, Hori M, Kitagawa M, Hasegawa T, et al. Influence of PD-L1 Expression in Immune Cells on the Response to Radiation Therapy in Patients With Oropharyngeal Squamous Cell Carcinoma. Radiotherapy Oncol (2018) 129(2):409-14. doi: 10.1016/j.radonc.2018.08.023

22. Hong AM, Ferguson P, Dodds T, Jones D, Li M, Yang J, et al. Significant Association of PD-L1 Expression With Human Papillomavirus Positivity and Its Prognostic Impact in Oropharyngeal Cancer. Oral Oncol (2019) 92:33-9. doi: 10.1016/j.oraloncology.2019.03.012

23. Hong AM, Vilain RE, Romanes S, Yang J, Smith E, Jones D, et al. PD-L1 Expression in Tonsillar Cancer Is Associated With Human Papillomavirus Positivity and Improved Survival: Implications for Anti-PD1 Clinical Trials. Oncotarget (2016) 7(47):77010-20. doi: 10.18632/oncotarget.12776

24. Kim HR, Ha S-J, Hong MH, Heo SJ, Koh YW, Choi EC, et al. PD-L1 Expression on Immune Cells, But Not on Tumor Cells, Is a Favorable Prognostic Factor for Head and Neck Cancer Patients. Sci Rep (2016) 6:36956-. doi: 10.1038/srep36956 
25. Kwon MJ, Rho Y-S, Nam ES, Cho SJ, Park H-R, Min SK, et al. Clinical Implication of Programmed Cell Death-1 Ligand-1 Expression in Tonsillar Squamous Cell Carcinoma in Association With Intratumoral Heterogeneity, Human Papillomavirus, and Epithelial-to-Mesenchymal Transition. Hum Pathol (2018) 80:28-39. doi: 10.1016/j.humpath.2018.03.025

26. Sato F, Ono T, Kawahara A, Kawaguchi T, Tanaka H, Shimamatsu K, et al. Prognostic Impact of P16 and PD-L1 Expression in Patients With Oropharyngeal Squamous Cell Carcinoma Receiving a Definitive Treatment. J Clin Pathol (2019) 72(8):542-9. doi: 10.1136/jclinpath-2019-205818

27. Solomon B, Young RJ, Bressel M, Urban D, Hendry S, Thai A, et al. Prognostic Significance of PD-L1(+) and CD8(+) Immune Cells in HPV(+) Oropharyngeal Squamous Cell Carcinoma. Cancer Immunol Res (2018) 6 (3):295-304. doi: 10.1158/2326-6066.CIR-17-0299

28. Balermpas P, Rödel F, Krause M, Linge A, Lohaus F, Baumann M, et al. The PD-1/PD-L1 Axis and Human Papilloma Virus in Patients With Head and Neck Cancer After Adjuvant Chemoradiotherapy: A Multicentre Study of the German Cancer Consortium Radiation Oncology Group (DKTK-ROG). Int J Cancer (2017) 141(3):594-603. doi: 10.1002/ijc.30770

29. Gurin D, Slavik M, Hermanova M, Selingerova I, Kazda T, Hendrych M, et al. The Tumor Immune Microenvironment and Its Implications for Clinical Outcome in Patients With Oropharyngeal Squamous Cell Carcinoma. J Oral Pathol Med (2020) 49(9):886-96. doi: 10.1111/jop.13055

30. Jeong JY, Park T-I, Ahn D. Comprehensive Analysis and Clinical Implication of PD-L1 Expression Considering HPV Status in Oropharyngeal Squamous Cell Carcinoma. Anticancer Res (2020) 40(7):4001-10. doi: 10.21873/anticanres.14394

31. Kim HS, Lee JY, Lim SH, Park K, Sun JM, Ko YH, et al. Association Between PD-L1 and HPV Status and the Prognostic Value of PD-L1 in Oropharyngeal Squamous Cell Carcinoma. Cancer Res Treat (2016) 48(2):527-36. doi: $10.4143 /$ crt.2015.249

32. Lilja-Fischer JK, Eriksen JG, Georgsen JB, Vo TT, Larsen SR, Cheng J, et al. Prognostic Impact of PD-L1 in Oropharyngeal Cancer After Primary Curative Radiotherapy and Relation to HPV and Tobacco Smoking. Acta Oncol (2020) 59(6):666-72. doi: 10.1080/0284186X.2020.1729407

33. Schoenfeld JD, Gjini E, Rodig SJ, Tishler RB, Rawal B, Catalano PJ, et al. Evaluating the PD-1 Axis and Immune Effector Cell Infiltration in Oropharyngeal Squamous Cell Carcinoma. Int J Radiat Oncology Biol Phys (2018) 102(1):137-45. doi: 10.1016/j.ijrobp.2018.05.002

34. Steuer CE, Griffith CC, Nannapaneni S, Patel MR, Liu Y, Magliocca KR, et al. A Correlative Analysis of PD-L1, PD-1, PD-L2, EGFR, HER2, and HER3 Expression in Oropharyngeal Squamous Cell Carcinoma. Mol Cancer Ther (2018) 17(3):710-6. doi: 10.1158/1535-7163.MCT-17-0504

35. Wuerdemann N, Gültekin SE, Pütz K, Wittekindt C, Huebbers CU, Sharma SJ, et al. PD-L1 Expression and a High Tumor Infiltrate of CD8+ Lymphocytes Predict Outcome in Patients With Oropharyngeal Squamous Cells Carcinoma. Int J Mol Sci (2020) 21(15):5228. doi: 10.3390/ijms21155228

36. Moher D, Shamseer L, Clarke M, Ghersi D, Liberati A, Petticrew M, et al. Preferred Reporting Items for Systematic Review and Meta-Analysis Protocols (PRISMA-P) 2015 Statement. Syst Rev (2015) 4(1):1. doi: 10.1186/2046-4053-4-1

37. Wells G, Shea B, O'Connell D, Peterson J, Welch V, Losos M, et al. The Newcastle-Ottawa Scale (NOS) for Assessing the Quality of Nonrandomised Studies in Meta-Analyses. Ottawa, CA: University of Ottawa (2014). Available at: http://www.ohri.ca/programs/clinical_epidemiology/oxford.asp.

38. Normand SL. Meta-Analysis: Formulating, Evaluating, Combining, and Reporting. Stat Med (1999) 18(3):321-59. doi: 10.1002/(SICI)1097-0258 (19990215)18:3<321::AID-SIM28>3.0.CO;2-P

39. Sterne JA, Egger M. Funnel Plots for Detecting Bias in Meta-Analysis: Guidelines on Choice of Axis. J Clin Epidemiol (2001) 54(10):1046-55. doi: 10.1016/S0895-4356(01)00377-8

40. De Meulenaere A, Vermassen T, Aspeslagh S, Huvenne W, Van Dorpe J, Ferdinande L, et al. Turning the Tide: Clinical Utility of PD-L1 Expression in Squamous Cell Carcinoma of the Head and Neck. Oral Oncol (2017) 70:34-42. doi: 10.1016/j.oraloncology.2017.05.002

41. Qiao X-w, Jiang J, Pang X, Huang M-c, Tang Y-j, Liang X-h, et al. The Evolving Landscape of PD-1/PD-L1 Pathway in Head and Neck Cancer. Front Immunol (2020) 11(1721). doi: 10.3389/fimmu.2020.01721

42. Li D, Yuan X, Liu J, Li C, Li W. Prognostic Value of Prognostic Nutritional Index in Lung Cancer: A Meta-Analysis. J Thorac Dis (2018) 10(9):5298-307. doi: $10.21037 /$ jtd.2018.08.51
43. Yang W-f, Wong MCM, Thomson PJ, Li K-Y, Su Y-x. The Prognostic Role of PD-L1 Expression for Survival in Head and Neck Squamous Cell Carcinoma: A Systematic Review and Meta-Analysis. Oral Oncol (2018) 86:81-90. doi: 10.1016/j.oraloncology.2018.09.016

44. Li J, Wang P, Xu Y. Prognostic Value of Programmed Cell Death Ligand 1 Expression in Patients With Head and Neck Cancer: A Systematic Review and Meta-Analysis. PloS One (2017) 12(6):e0179536. doi: 10.1371/journal.pone. 0179536

45. Taube JM, Anders RA, Young GD, Xu H, Sharma R, McMiller TL, et al. Colocalization of Inflammatory Response With B7-H1 Expression in Human Melanocytic Lesions Supports an Adaptive Resistance Mechanism of Immune Escape. Sci Trans Med (2012) 4(127):127ra37. doi: 10.1126/scitranslmed. 3003689

46. Droeser RA, Hirt C, Viehl CT, Frey DM, Nebiker C, Huber X, et al. Clinical Impact of Programmed Cell Death Ligand 1 Expression in Colorectal Cancer. Eur J Cancer (2013) 49(9):2233-42. doi: 10.1016/j.ejca.2013.02.015

47. Lipson EJ, Vincent JG, Loyo M, Kagohara LT, Luber BS, Wang H, et al. PD-L1 Expression in the Merkel Cell Carcinoma Microenvironment: Association With Inflammation, Merkel Cell Polyomavirus and Overall Survival. Cancer Immunol Res (2013) 1(1):54-63. doi: 10.1158/2326-6066. CIR-13-0034

48. Liu J, Liu Y, Wang W, Wang C, Che Y. Expression of Immune Checkpoint Molecules in Endometrial Carcinoma. Exp Ther Med (2015) 10(5):1947-52. doi: 10.3892/etm.2015.2714

49. Xu D, Dong P, Xiong Y, Chen R, Konno Y, Ihira K, et al. PD-L1 Is a Tumor Suppressor in Aggressive Endometrial Cancer Cells and Its Expression Is Regulated by miR-216a and IncRNA Meg3. Front Cell Dev Biol (2020) 8 (1488). doi: 10.3389/fcell.2020.598205

50. Allouch S, Malki A, Allouch A, Gupta I, Vranic S, Al Moustafa A-E. High-Risk HPV Oncoproteins and PD-1/PD-L1 Interplay in Human Cervical Cancer: Recent Evidence and Future Directions. Front Oncol (2020) 10(914). doi: 10.3389/fonc. 2020.00914

51. De Keukeleire SJ, Vermassen T, Hilgert E, Creytens D, Ferdinande L, Rottey S. Immuno-Oncological Biomarkers for Squamous Cell Cancer of the Head and Neck: Current State of the Art and Future Perspectives. Cancers (2021) 13 (7):1714. doi: 10.3390/cancers13071714

52. Stern PL, Dalianis T. Oropharyngeal Squamous Cell Carcinoma Treatment in the Era of Immune Checkpoint Inhibitors. Viruses (2021) 13(7). doi: 10.3390/ v13071234

53. Cristina V, Herrera-Gómez RG, Szturz P, Espeli V, Siano M. Immunotherapies and Future Combination Strategies for Head and Neck Squamous Cell Carcinoma. Int J Mol Sci (2019) 20(21). doi: 10.3390/ ijms20215399

54. Ang KK, Harris J, Wheeler R, Weber R, Rosenthal DI, Nguyen-Tan PF, et al. Human Papillomavirus and Survival of Patients With Oropharyngeal Cancer. N Engl J Med (2010) 363(1):24-35. doi: 10.1056/ NEJMoa0912217

55. Gillison ML, Trotti AM, Harris J, Eisbruch A, Harari PM, Adelstein DJ, et al. Radiotherapy Plus Cetuximab or Cisplatin in Human Papillomavirus-Positive Oropharyngeal Cancer (NRG Oncology RTOG 1016): A Randomised, Multicentre, Non-Inferiority Trial. Lancet (2019) 393(10166):40-50. doi: 10.1016/S0140-6736(18)32779-X

56. Mehanna H, Robinson M, Hartley A, Kong A, Foran B, Fulton-Lieuw T, et al. Radiotherapy Plus Cisplatin or Cetuximab in Low-Risk Human Papillomavirus-Positive Oropharyngeal Cancer (De-ESCALaTE HPV): An Open-Label Randomised Controlled Phase 3 Trial. Lancet (2019) 393 (10166):51-60. doi: 10.1016/S0140-6736(18)32752-1

57. Fialová A, Koucký V, Hajdušková M, Hladíková K, Špíšek R. Immunological Network in Head and Neck Squamous Cell Carcinoma-A Prognostic Tool Beyond HPV Status. Front Oncol (2020) 10(1701). doi: 10.3389/fonc. 2020.01701

58. von Hippel PT. The Heterogeneity Statistic I(2) can be Biased in Small MetaAnalyses. BMC Med Res Methodol (2015) 15:35. doi: 10.1186/s12874-0150024-Z

59. Zou Y, Hu X, Zheng S, Yang A, Li X, Tang H, et al. Discordance of Immunotherapy Response Predictive Biomarkers Between Primary Lesions and Paired Metastases in Tumours: A Systematic Review and Meta-Analysis. EBioMedicine (2021) 63. doi: 10.1016/j.ebiom.2020.103137 
60. Poropatich K, Hernandez D, Fontanarosa J, Brown K, Woloschak G, Paintal A, et al. Peritumoral Cuffing by T-Cell Tumor-Infiltrating Lymphocytes Distinguishes HPV-Related Oropharyngeal Squamous Cell Carcinoma From Oral Cavity Squamous Cell Carcinoma. J Oral Pathol Med (2017) 46(10):972-8. doi: 10.1111/jop.12605

Conflict of Interest: The authors declare that the research was conducted in the absence of any commercial or financial relationships that could be construed as a potential conflict of interest.

Publisher's Note: All claims expressed in this article are solely those of the authors and do not necessarily represent those of their affiliated organizations, or those of the publisher, the editors and the reviewers. Any product that may be evaluated in this article, or claim that may be made by its manufacturer, is not guaranteed or endorsed by the publisher.

Copyright () 2021 Polesel, Menegaldo, Tirelli, Giacomarra, Guerrieri, Baboci, Casarotto, Lupato, Fanetti, Boscolo-Rizzo and Fratta. This is an open-access article distributed under the terms of the Creative Commons Attribution License (CC BY). The use, distribution or reproduction in other forums is permitted, provided the original author(s) and the copyright owner(s) are credited and that the original publication in this journal is cited, in accordance with accepted academic practice. No use, distribution or reproduction is permitted which does not comply with these terms. 